\title{
Idiopathic Pulmonary Fibrosis and Emphysema: Between Scylla and Charybdis
}

\author{
Vasilios Tzilas ${ }^{\mathrm{a}}$ Argyris Tzouvelekis ${ }^{\mathrm{a}}$ Spyridon Papiris ${ }^{\mathrm{b}}$ Demosthenes Bouros ${ }^{\mathrm{a}}$ \\ a 1st Respiratory Medicine Department, Hospital for Diseases of the Chest 'Sotiria', and ' 2nd Respiratory Medicine \\ Department, 'Attikon' University Hospital, Athens Medical School, National and Kapodistrian University of Athens, \\ Athens, Greece
}

Idiopathic pulmonary fibrosis (IPF) is the commonest of the idiopathic interstitial pneumonias and carries the most ominous prognosis with a median survival of 3 years [1]. IPF often coexists with emphysema. The coexistence of IPF and emphysema is intriguing as they share common pathogenetic mechanisms [2] and alongside lung cancer they have been considered as different manifestations of smoking on a susceptible genetic background [3]. But also from a clinical view the coexistence of IPF and emphysema is very interesting [4]. Firstly, it represents a characteristic case in which the presence of slightly subnormal or even normal forced vital capacity does not exclude the possibility of underlying fibrosis. Secondly, it has opened the question of whether combined pulmonary fibrosis and emphysema (CPFE) patients exhibit worse survival in comparison to sole IPF patients. Literature so far has exhibited contradicting results regarding the survival of CPFE versus sole IPF patients with some of the studies showing worsened survival $[5,6]$ and other studies showing no difference $[7,8]$ or even improved survival $[9,10]$. Several reasons could be implicated for the discrepant results of the studies so far [11-13]. One of these reasons could be the inclusion in the pulmonary fibrosis group of patients with nonspe- cific interstitial pneumonia, which has a better prognosis compared to IPF.

In this issue of Respiration, Kohashi et al. [14] add further information on the impact of emphysema on IPF prognosis. The novelty of their study lies in the fact that they strictly included patients with biopsy-proven IPF. A total of 47 patients with biopsy-proven IPF were analyzed. For the assessment of emphysema and fibrosis, high-resolution computed tomography scans were evaluated semiquantitatively by visual scoring. The lungs were divided into six zones (upper, middle, and lower zones bilaterally) and each zone was evaluated separately according to the following: score $0=$ none; score $0.5=<5 \%$; score $1=$ $5-24 \%$; score $2=25-49 \%$; score $3=50-74 \%$, and score $4=\leq 75 \%$. IPF-emphysema was defined as IPF cases with a total emphysema score greater than 3.0, with mean scores of all 6 zones greater than 0.50. Eight patients fulfilled the previous criteria forming the group of IPF-emphysema. Patients with IPF-emphysema had a statistically significantly worst survival compared to IPF alone patients by Kaplan-Meier analysis (median survival 1,734 vs. 2,229 days, respectively, $\mathrm{p}=0.007)$. As expected, emphysema had upper lobe predominance. Regarding other prognostic parameters on univariate analysis, Krebs von

\section{KARGER}

E-Mail karger@karger.com

www.karger.com/res
C2016 S. Karger AG, Basel

0025-7931/16/0924-0215\$39.50/0
Prof. Demosthenes Bouros, MD, PhD, FERS, FCCP, FAPSR

Medical School, University of Athens, Academic Department of Pneumonology Hospital for Diseases of the Chest 'Sotiria'

Messogion Ave. 152, GR-11527 Athens (Greece)

E-Mail dbouros@med.uoa.gr 
den Lungen-6, surfactant protein-D, $\mathrm{PaO}_{2}, \mathrm{FVC}$, DLCO and total emphysema score $>3.0$ were poor prognosticators, while on multivariate analysis DLCO and total emphysema score $>3.0$ retained their statistical significance. Interestingly, none of the patients in the study was diagnosed with lung cancer while in other studies, CPFE constituted a stronger predictor of the occurrence of lung cancer in relation to emphysema [15-17]. Also, in contrast to previous studies $[5,6,18,19]$, the presence of pulmonary hypertension (assessed by cardiac ultrasonography) was no significantly different between the two groups.

The main limitation of the study is the low number of patients (47 IPF patients with 8 patients fulfilling the criteria to enter the IPF-emphysema group). Its retrospective nature is another limitation. Also, we must keep in mind that visual scoring of emphysema and fibrosis is an extremely difficult task, especially in CPFE patients. In the latter group, interobserver agreement on scoring can be poor even amongst experienced thoracic radiologists. The distinction between emphysema and fibrosis on high-resolution computed tomography is expected to be problematic as these two entities are overlapping even on pathology level [20]. Finally, the type of emphysema (paraseptal vs. centrilobular) was not studied. This can be clinically important as paraseptal emphysema has been related to a higher extent of fibrosis [21]. Strengths of the study are not only the fact that IPF diagnosis was biopsy proven but also that diagnosis was cemented within the context of multidisciplinary discussion between respiratory physicians, thoracic radiologists and pulmonary pathologists.
It is important to stress that regarding emphysema scoring, a threshold of 3.0 was chosen to differentiate IPFemphysema from IPF alone patients, meaning that some IPF alone patients did have some degree of emphysema. Prognosis did not differ between patients with sole IPF and patients with IPF and emphysema in general (i.e. score $>0$ ). It is also very interesting that even when this threshold was only slightly reduced to 2.5 , the survival between IPF-emphysema and IPF alone patients was not statistically significant $(\mathrm{p}=0.345)$. This observation is very important as it lets us consider that the clinical impact of emphysema in the described group of patients was related to its extent. Thus, prognosis seems to be not just a matter of coexisting emphysema but also of 'how much' emphysema. Expectedly, DLCO, which is influenced by both fibrosis and emphysema and reflects the functional result of these two conditions, was the one of the two significant prognostic parameters (the other being emphysema score $>3.0$ ) in multivariate analysis. Emphysema and fibrosis scores were as expected significantly correlated with DLCO. In the studies by Ryerson et al. [8] and Kurashima et al. [9], a threshold of $10 \%$ was used to separate sole IPF from CPFE patients. Common sense dictates that in a fibrotic lung, further loss of functional lung parenchyma due to emphysema represents an additional aggravating factor. In order to clarify the impact of emphysema on the survival of CPFE, it is crucial not only to define the kind of underlying fibrosis but also to establish universally accepted criteria on the extent of clinically meaningful emphysema.

\section{References}

1 Raghu G, et al: An official ATS/ERS/JRS/ ALAT statement: idiopathic pulmonary fibrosis: evidence-based guidelines for diagnosis and management. Am J Respir Crit Care Med 2011;183:788-824.

2 Tzilas V, Bouros D: Pathogenesis of combined pulmonary fibrosis and emphysema. Common pathogenetic pathways. Pneumon 2015;28:133-138.

-3 Chilosi M, Poletti V, Rossi A: The pathogenesis of COPD and IPF: distinct horns of the same devil? Respir Res 2012;13:3.

-4 Papaioannou AI, Kostikas K, Manali ED, et al: Combined pulmonary fibrosis and emphysema: the many aspects of a cohabitation contract. Respir Med 2016;117:14-26.
Mejia M, Carrillo G, Rojas-Serrano J, et al: Idiopathic pulmonary fibrosis and emphysema: decreased survival associated with severe pulmonary arterial hypertension. Chest 2009; 136:10-15.

-6 Sugino K, Ishida F, Kikuchi N, et al: Comparison of clinical characteristics and prognostic factors of combined pulmonary fibrosis and emphysema versus idiopathic pulmonary fibrosis alone. Respirology 2014;19:239-245.

7 Jankowich MD, Rounds S: Combined pulmonary fibrosis and emphysema alters physiology but has similar mortality to pulmonary fibrosis without emphysema. Lung 2010;188: 365-373.

-8 Ryerson CJ, Hartman T, Elicker BM, et al: Clinical features and outcomes in combined pulmonary fibrosis and emphysema in idiopathic pulmonary fibrosis. Chest 2013;144: 234-240.
Kurashima K, Takayanagi N, Tsuchiya N, et al: The effect of emphysema on lung function and survival in patients with idiopathic pulmonar fibrosis. Respirology 2010;15:843848.

10 Todd NW, Jeudy J, Lavania S, et al: Centrilobular emphysema combined with pulmonary fibrosis results in improved survival. Fibrogenesis Tissue Repair 2011;4:6.

-11 Papiris SA, Triantafillidou C, Manali ED, et al: Combined pulmonary fibrosis and emphysema. Expert Rev Respir Med 2013;7:19-31.

$\checkmark 12$ Papaioannou AI, Kostikas K, Manali ED, et al: Serum Levels of Surfactant Proteins in Patients with Combined Pulmonary Fibrosis and Emphysema (CPFE). PLoS One 2016; 11:e0157789. 
13 Tzilas V, Bouros D: Combined pulmonary fibrosis and emphysema: a clinical review. COPD Res Pract 2016;2:2.

14 Kohashi Y, Arai T, Sugimoto C, et al: Clinical impact of emphysema evaluated by high-resolution computed tomography on idiopathic pulmonary fibrosis diagnosed by surgical lung biopsy. Respiration 2016;92:220-228.

15 Kwak N, Park CM, Lee J, et al: Lung cancer risk among patients with combined pulmonary fibrosis and emphysema. Respir Med 2014;108:524.e30.
16 Usui K, Tanai C, Tanaka Y, Noda H, Ishihara $\mathrm{T}$ : The prevalence of pulmonary fibrosis combined with emphysema in patients with lung cancer. Respirology 2011;16:326-331.

17 Girard N, Marchand-Adam S, Naccache JM, et al; Groupe d'Etudes et de Recherche sur les Maladies 'Orphelines' Pulmonaires (GERM'O'P): Lung cancer in combined pulmonary fibrosis and emphysema: a series of 47 Western patients. J Thorac Oncol 2014;9: $1162-1170$.

18 Cottin V, Nunes H, Brillet P, et al: Combined pulmonary fibrosis and emphysema: a distinct underrecognised entity. Eur Respir J 2005;26:586-593.
9 Cottin V, Le Pavec J, Prévot G, et al: Pulmonary hypertension in patients with combined pulmonary fibrosis and emphysema syndrome. Eur Respir J 2010;35:105-111.

20 Inomata M, Ikushima S, Awano N, et al: An autopsy study of combined pulmonary fibrosis and emphysema: correlations among clinical, radiological, and pathological features. BMC Pulm Med 2014;14:104.

21 Oikonomou A, Mintzopoulou P, Tzouvelekis A, et al: Pulmonary fibrosis and emphysema: Is the emphysema type associated with the pattern of fibrosis? World J Radiol 2015;7: 294-305. 